\title{
How disorder affects the Berry-phase anomalous Hall conductivity: A reciprocal-space analysis
}

\author{
Raffaello Bianco, ${ }^{1,2}$ Raffaele Resta, ${ }^{1,3}$ and Ivo Souza ${ }^{2,4}$ \\ ${ }^{1}$ Dipartimento di Fisica, Università di Trieste, 34127 Trieste, Italy \\ ${ }^{2}$ Centro de Física de Materiales, Universidad del País Vasco, 20018 San Sebastián, Spain \\ ${ }^{3}$ Donostia International Physics Center, 20018 San Sebastián, Spain \\ ${ }^{4}$ Ikerbasque Foundation, 48013 Bilbao, Spain
}

(Received 14 April 2014; revised manuscript received 16 August 2014; published 29 September 2014)

\begin{abstract}
The anomalous Hall conductivity of "dirty" ferromagnetic metals is dominated by a Berry-phase contribution which is usually interpreted as an intrinsic property of the Bloch electrons in the pristine crystal. In this work we evaluate the geometric Hall current directly from the electronic ground state with disorder and then recast it as an integral over the crystalline Brillouin zone. The integrand is an effective $k$-space Berry curvature, obtained by unfolding the Berry curvature from the small Brillouin zone of a large supercell. Therein, disorder yields a net extrinsic Hall contribution, which we argue is related to the elusive side-jump effect. As an example, we unfold the first-principles Berry curvature of an ordered $\mathrm{Fe}_{3} \mathrm{Co}$ alloy from the original fcc-lattice Brillouin zone onto a bcc-lattice zone with four times the volume. Comparison with the virtual-crystal Berry curvature clearly reveals the symmetry-breaking effects of the substitutional Co atoms.
\end{abstract}

DOI: 10.1103/PhysRevB.90.125153

PACS number(s): 72.15.Eb, 72.20.Dp, 72.20.My, 72.25.-b

\section{INTRODUCTION}

The anomalous Hall effect (AHE) in ferromagnetic metals includes a band-structure contribution given by the $k$-space Berry curvature of the occupied Bloch states [1,2]. Somewhat counterintuitively, this intrinsic contribution only becomes dominant in moderately resistive ("dirty") samples, for which crystal momentum is not a good quantum number and the Berry curvature strictly speaking is ill defined. In highly conducting pristine samples with sharply defined energy bands and Berry curvature, the AHE is instead dominated by an extrinsic contribution unrelated to the Berry curvature: skew scattering from dilute impurities [1].

Motivated by these considerations, we introduce an effective $k$-space Berry curvature which remains well defined in the presence of certain types of disorder, namely, those types which are describable by reference to an underlying ordered lattice. Examples of such "cellular disorder" include chemical disorder, substitutional or interstitial impurities, vacancies, and thermal disorder of the atomic positions and spin orientations [3].

The effective Berry curvature is defined in the Brillouin zone (BZ) of the reference lattice in such a way that its BZ integral gives the anomalous Hall conductivity (AHC) as a property of the disordered electronic ground state. We call this the geometric AHC contribution: It combines the nominally intrinsic contribution with certain disorder effects of a similar nature. With this definition we depart from the standard terminology, where the terms "intrinsic" and "geometric" (or "Berry-phase") are used interchangeably when referring to AHC contributions [1,2]. The proposed definition should be particularly useful for studying the AHC of magnetic alloys with well-characterized chemical disorder.

We begin by noting that in supercell (SC) calculations a disordered system is treated as a "crystal" with the artificial SC periodicity. Its eigenstates are formally Bloch states, labeled by a wave vector in the SC BZ. Hence, the AHC can be cast in the same geometric form as the intrinsic $\mathrm{AHC}$ of an actual crystal: a Berry curvature summed over the occupied states and integrated over the (folded) BZ. Specializing to cellular disorder, we then use "BZ unfolding" techniques [4-7] to map the SC Berry curvature onto the BZ of the reference lattice. This procedure gives the effective $k$-space Berry curvature, henceforth denoted unfolded Berry curvature.

We emphasize that the total geometric AHC is not affected by the unfolding procedure [Eqs. (4) and (5) below]; what the unfolded Berry curvature provides is the detailed $k$-space distribution of the AHC, including disorder contributions. This is particularly valuable, given that the (genuinely) intrinsic AHC is strongly influenced by sharp features in the Berry curvature of the pristine crystal [8-10].

We demonstrate our approach by analyzing the effect of the impurities on a very crude model of a disordered magnetic alloy: a regular $\mathrm{Fe}_{3} \mathrm{Co}$ crystal. By means of the unfolded curvature, we visualize the effects of scattering (in our case by $\mathrm{Co}$ ) as due to reciprocal-space couplings. Scattering effects are instead completely washed out within a standard virtual-crystal approximation (VCA), which can be regarded as the "clean limit" of a fictitious crystal. While the present implementation is first principles, our calculations are intended to be illustrative only and do not aim to provide accurate results for the AHC of real Fe-Co alloys [11]; more realistic calculations would require larger $\mathrm{SCs}$, lattice-constant optimization, and disorder averaging.

The paper is organized as follows. In Sec. II we outline the well-established theory of the intrinsic AHC, as well as our approach to extend it in order to to include, on the same footing, some effects of disorder. In Sec. III we motivate our approach starting from the Berry curvature defined in the folded BZ of a disordered SC. In Sec. IV we introduce a general BZ unfolding formalism, which we then apply to the Berry curvature; the details of the implementation in a Wannier-function basis are also given. In Sec. VI we compute from first principles the unfolded curvature of our model $\mathrm{Fe}-\mathrm{Co}$ alloy and compare it with the Berry curvatures of pure bcc Fe and of the alloy in the VCA. We conclude in Sec. VII with a discussion and an outlook. 


\section{INTRINSIC VS EXTRINSIC}

Our generalized Berry-curvature definition in the presence of disorder is based on the notion of BZ unfolding, which has been used extensively in recent years in the context of band-structure calculations with periodic SCs [4-7]. Similar unfolding techniques were introduced long ago to describe the phonon spectra of disordered alloys [12].

Unfolding band structures of SC calculations is a particularly informative way of visualizing the influence of cellular disorder on the electronic states. For weak to moderate disorder the unfolded bands resemble those of the pristine (or virtual) crystal, with the deviations in both the dispersion and the spectral weight reflecting the effect of the disorder potential [4]. The recent development of efficient $a b$ initiobased SC methodologies [13] opens up new possibilities for applying unfolding techniques to large SCs with realistic descriptions of disorder $[14,15]$.

So far, BZ unfolding has been used mainly to extract approximate energy dispersions for disordered systems. While the energy bands $\epsilon_{\mathbf{k} i}$ are the most basic quantity in the theory of solids, it is now understood that the $k$-space Berry curvature $\boldsymbol{\Omega}_{i}(\mathbf{k})=\nabla_{\mathbf{k}} \times \mathbf{A}_{i}(\mathbf{k})$ is an additional fundamental ingredient determining the dynamics of electrons in crystals [2]. [Here $\mathbf{A}_{i}(\mathbf{k})$ is the Berry connection, to be defined shortly.] Using Stokes' theorem, the Berry curvature can be viewed as the geometric phase $\varphi_{i}=\oint \mathbf{A}_{i}(\mathbf{k}) \cdot d \mathbf{l}$ per unit area picked up by a Bloch electron in band $i$ as it is transported adiabatically along a small loop in $k$ space. The Berry curvature is generically nonzero in the BZ of crystals with broken inversion or timereversal symmetry. It modifies the motion of electron wave packets driven by an electric field $\boldsymbol{E}$ by adding a transverse "anomalous velocity" term $(e / \hbar) \boldsymbol{\Omega}_{i}(\mathbf{k}) \times \boldsymbol{E}$ to the usual band velocity $(1 / \hbar) \nabla_{\mathbf{k}} \epsilon_{\mathbf{k} j}$.

The intrinsic $\mathrm{AHC}$ is a direct consequence of the anomalous velocity. It is given by $[1,2]$

$$
\begin{gathered}
\sigma_{a b}^{\mathrm{int}}=-\frac{e^{2}}{\hbar} \int_{\mathrm{NBZ}} \frac{d^{3} k}{(2 \pi)^{3}} \Omega_{a b}^{\mathrm{occ}}(\mathbf{k}), \\
\Omega_{a b}^{\mathrm{occ}}(\mathbf{k})=\sum_{i} f_{\mathbf{k} i} \Omega_{i, a b}(\mathbf{k}), \\
\Omega_{i, a b}(\mathbf{k})=\epsilon_{a b c} \Omega_{i, c}(\mathbf{k})=\nabla_{k_{a}} A_{i, b}(\mathbf{k})-\nabla_{k_{b}} A_{i, a}(\mathbf{k}),
\end{gathered}
$$

where $f_{\mathbf{k} i}$ is the occupation of the Bloch eigenstate $|\mathbf{k} i\rangle=$ $e^{i \mathbf{k} \cdot \hat{\mathbf{x}}}\left|u_{\mathbf{k} i}\right\rangle$ and $\mathbf{A}_{i}(\mathbf{k})=i\left\langle u_{\mathbf{k} i} \mid \nabla_{\mathbf{k}} u_{\mathbf{k} i}\right\rangle$ is the Berry connection of the $i$ th band. The integral in Eq. (1) is over the BZ of the pristine crystal, which we call the normal Brillouin zone (NBZ).

The definition of the intrinsic AHC as a Berry curvature in $k$ space relies on perfect translational order. This is at odds with the above-mentioned fact that the intrinsic contribution tends to dominate in dirty samples, where translational order is significantly disrupted. The conventional formulation becomes even more problematic for intrinsically disordered systems such as random alloys, for which there is no experimentally accessible clean limit. Yet, it is still useful to reason in terms of "intrinsic" contributions to the AHE in such materials [16].

In view of these difficulties, how should the intrinsic AHC be defined and calculated in the presence of disorder? The standard procedure is to define it in terms of the Berry curvature of an ordered reference system - the pristine crystal in the case of doped samples $[8,17]$ or a virtual-crystal effective Hamiltonian in the case of alloys $[18,19]$ — calculated using the band filling appropriate to the doping level or alloying concentration. In the case of alloys, disorder effects are sometimes included via a diagonal self-energy term $\eta^{2}$ in the sum-over-states expression for the (virtual-crystal) Berry curvature [see Eq. (6) below] to account for the finite lifetime of the Bloch eigenstates [17]. A related strategy, which has been implemented within the coherent-potential approximation, is to compute the intrinsic AHC of the alloy starting from the Kubo-Strda equation, by combining all terms not connected to vertex corrections [16]. While physically motivated, these remain somewhat ad hoc and model-dependent prescriptions, which can only be justified for sufficiently dilute or concentrated alloys.

We propose a different approach, where we do not insist on defining precisely the intrinsic AHC contribution in a disordered system and focus instead on the geometric AHC. In the SC approach it is computed by inserting into Eq. (1) the electronic states of the SC system,

$$
\sigma_{a b}^{\mathrm{geom}}=-\frac{e^{2}}{\hbar} \int_{\mathrm{SBZ}} \frac{d^{3} K}{(2 \pi)^{3}} \Omega_{a b}^{\mathrm{occ}}(\mathbf{K}),
$$

and averaging over several realizations of disorder. Here $\Omega_{a b}^{\text {occ }}(\mathbf{K})$ is the Berry curvature of the occupied SC eigenstates $|\mathbf{K} J\rangle$ and the integral is over the SC Brillouin zone (SBZ). No phenomenological lifetime broadening parameter needs to be included in the Berry curvature because spectral broadening by disorder is already built in, as revealed by the configurationaveraged unfolded bands [13-15].

Equation (4) correctly gives a quantized value for the AHC of two-dimensional disordered Chern insulators when the Fermi level lies in the mobility gap [20,21], and we propose to use it to unambiguously identify a dominant contribution to the AHC of metallic disordered systems. [Contrary to the case of Chern insulators, Eq. (4) may not capture the full AHC of disordered metals in SC calculations; we return to this point in Sec. VII.]

Realistic descriptions of disorder require reasonably large SCs. The integration volume in Eq. (4) then becomes very small, and all $k$-space information is lost. In order to restore a $k$-space description reminiscent of Eq. (1), we recast Eq. (4) as the NBZ integral of the unfolded Berry curvature $\boldsymbol{\Omega}^{\text {unf }}(\mathbf{k})$, to be defined precisely in Sec. IV:

$$
\sigma_{a b}^{\mathrm{geom}}=-\frac{e^{2}}{\hbar} \int_{\mathrm{NBZ}} \frac{d^{3} k}{(2 \pi)^{3}} \Omega_{a b}^{\mathrm{unf}}(\mathbf{k}) .
$$

Although with disorder present the unfolded curvature is no longer geometric in the NBZ (the interpretation as a Berry phase per unit area is lost), it remains gauge invariant.

\section{BERRY CURVATURE IN THE FOLDED BRILLOUIN ZONE}

Starting from the Kubo-Greenwood formula for the AHC, one finds for the quantity $\Omega_{a b}^{\text {occ }}(\mathbf{k})$ in Eq. (1)

$$
\Omega_{a b}^{\mathrm{occ}}(\mathbf{k})=\operatorname{Im} \sum_{i, j}\left(f_{\mathbf{k} j}-f_{\mathbf{k} i}\right) \frac{\left\langle\mathbf{k} i\left|\hbar \hat{v}_{a}\right| \mathbf{k} j\right\rangle\left\langle\mathbf{k} j\left|\hbar \hat{v}_{b}\right| \mathbf{k} i\right\rangle}{\left(\epsilon_{\mathbf{k} j}-\epsilon_{\mathbf{k} i}\right)^{2}+\eta^{2}} .
$$


In the limit of $\eta \rightarrow 0^{+}$this expression can be recast in the geometric form of Eq. (2) [1].

Equation (6) is written in terms of the Bloch eigenstates and energy eigenvalues of a pristine crystal. If we place the crystal in a periodic SC and introduce some disorder, the disorder potential mixes states with different NBZ momenta $\mathbf{k}$ and $\mathbf{k}^{\prime}$ whenever $\mathbf{k}^{\prime}-\mathbf{k}$ equals a SC reciprocal vector $\mathbf{G}$, forcing the new eigenstates to be labeled by a common wave vector $\mathbf{K}$ in the SBZ. The Berry curvature can still be defined in the SBZ from Eq. (6), now written in terms of the SC eigenstates $|\mathbf{K} J\rangle$, energy eigenvalues $\epsilon_{\mathbf{K} J}$, and occupations $f_{\mathbf{K} J}$. Upon setting $\eta=0$ this leads to Eq. (4) for the geometric AHC.

Because of those extra couplings from disorder, it is not obvious how to map (unfold) the Berry curvature from the SBZ onto the NBZ of the original crystal. Clearly, $\boldsymbol{\Omega}^{\text {occ }}(\mathbf{K})$ is not simply equal to the virtual-crystal Berry curvature summed over the points $\left\{\mathbf{k}_{s}\right\}$ which fold onto $\mathbf{K}: \boldsymbol{\Omega}^{\text {occ }}(\mathbf{K}) \neq$ $\sum_{s} \boldsymbol{\Omega}_{\mathrm{VCA}}^{\mathrm{occ}}\left(\mathbf{k}_{s}\right)$. Nevertheless, it will be possible, for cellular disorder, to arrive at a unique definition for the unfolded Berry curvature with all the desired properties, namely: (i) it reduces to the ordinary Berry curvature $\boldsymbol{\Omega}^{\mathrm{occ}}(\mathbf{k})$ in the disorder-free limit; (ii) it remains sharply defined (gauge invariant) in the presence of disorder; and (iii) it constitutes a proper mapping from the SBZ to the NBZ in the sense that

$$
\boldsymbol{\Omega}^{\mathrm{occ}}(\mathbf{K})=\sum_{s} \boldsymbol{\Omega}^{\mathrm{unf}}\left(\mathbf{k}_{s}\right)
$$

which provides the link between Eqs. (4) and (5).

The difference between the unfolded SC curvature and the Berry curvature of the virtual crystal with averaged disorder reflects the disorder-mediated couplings between the folded bands, made possible by the relaxed crystal-momentum selection rule inside the SC. Such "pseudodirect" transitions [6] modify the interband coherence effects described by Eq. (6), giving additional contributions to the anomalous velocity and AHC which are absorbed into the definition of $\boldsymbol{\Omega}^{\text {unf }}(\mathbf{k})$.

A striking feature of the Berry curvature in crystalline ferromagnets is the occurrence of sharp peaks when two energy bands lying on either side of the Fermi level become quasidegenerate [8-10]. This can be understood in terms of Eq. (6) as a resonant enhancement behavior, and we see that the same intuitive picture holds for the unfolded quantities: Strong peaks in $\boldsymbol{\Omega}^{\text {unf }}(\mathbf{k})$ can be traced back to pairs of unfolded bands separated by small (pseudo)direct gaps across $\epsilon_{F}$.

\section{BRILLOUIN-ZONE UNFOLDING}

\section{A. Basic definitions}

Given a set of primitive translations $\left\{\mathbf{a}_{i}\right\}$ of the normal crystal cell (NC), the SC primitive translations can be written as $\sum_{j} M_{i j} \mathbf{a}_{j}$, with $\mathbf{M}$ an integer matrix. Each point $\mathbf{K}$ in the SBZ unfolds onto $|\mathbf{M}|$ distinct points $\mathbf{k}_{s}=\mathbf{K}+\mathbf{G}_{s}$ in the NBZ, where the $\mathbf{G}_{s}$ are SC reciprocal lattice vectors [6].

Following Refs. [4,5] we introduce a Bloch basis in the NBZ, and another in the SBZ. The basis states $|\mathbf{k} n\rangle$ and $|\mathbf{K} N\rangle$ are normalized over the $\mathrm{NC}$ and the $\mathrm{SC}$, respectively, and we define $\langle\cdots\rangle$ as an integral over the SC volume, so that $\left\langle\mathbf{k} n \mid \mathbf{k}^{\prime} m\right\rangle=|\mathbf{M}| \delta_{n, m} \delta_{\mathbf{k}, \mathbf{k}^{\prime}}$.
We also define the projection operator

$$
\hat{T}(\mathbf{k})=\frac{1}{|\mathbf{M}|} \sum_{n}|\mathbf{k} n\rangle\langle\mathbf{k} n|,
$$

such that

$$
\sum_{s} \hat{T}\left(\mathbf{k}_{s}\right)|\mathbf{K} N\rangle=|\mathbf{K} N\rangle .
$$

Thus, any SC Bloch state $|\mathbf{K} N\rangle$ has unfolded Bloch character distributed among the points $\left\{\mathbf{k}_{s}\right\}$, with weights $\left\langle\mathbf{K} N\left|\hat{T}\left(\mathbf{k}_{s}\right)\right| \mathbf{K} N\right\rangle$ which add up to 1 [7].

\section{B. Unfolding a generic $k$-space quantity}

Consider a property of the SC system which can be calculated by taking the trace of a Hermitian matrix,

$$
\mathcal{O}_{N M}(\mathbf{K})=\langle\mathbf{K} N|\hat{\mathcal{O}}| \mathbf{K} M\rangle,
$$

followed by an integration over the SBZ. Our goal is to recast that property as an integral over the NBZ, by mapping $\operatorname{Tr} \mathcal{O}(\mathbf{K})$ onto the NBZ. This can be achieved using Eq. (9) together with the completeness relation for the SC Bloch basis:

$$
\begin{aligned}
\operatorname{Tr} \mathcal{O}(\mathbf{K})= & \sum_{N}\langle\mathbf{K} N|\hat{\mathcal{O}}| \mathbf{K} N\rangle \\
= & \frac{1}{|\mathbf{M}|^{2}} \sum_{s, n}\left\langle\mathbf{k}_{s} n|\hat{\mathcal{O}}| \mathbf{k}_{t} m\right\rangle\left\langle\mathbf{k}_{t} m\right| \\
& \times\left[\sum_{t, m}|\mathbf{K} N\rangle\langle\mathbf{K} N|\right]\left|\mathbf{k}_{s} n\right\rangle \\
= & \frac{1}{|\mathbf{M}|^{2}} \sum_{s, n}\left\langle\mathbf{k}_{s} n|\hat{\mathcal{O}}| \mathbf{k}_{t} m\right\rangle\left\langle\mathbf{k}_{t} m \mid \mathbf{k}_{s} n\right\rangle \\
= & \sum_{s, n} \frac{1}{|\mathbf{M}|}\left\langle\mathbf{k}_{s} n|\hat{\mathcal{O}}| \mathbf{k}_{s} n\right\rangle .
\end{aligned}
$$

Note that off-diagonal terms with $t \neq s$ do not contribute. Defining the unfolded quantity $\mathcal{O}^{\text {unf }}\left(\mathbf{k}_{s}\right)$ as the trace of

$$
\mathcal{O}_{n m}\left(\mathbf{k}_{s}\right)=\frac{1}{|\mathbf{M}|}\left\langle\mathbf{k}_{s} n|\hat{\mathcal{O}}| \mathbf{k}_{s} m\right\rangle
$$

Eq. (11) becomes

$$
\operatorname{Tr} \mathcal{O}(\mathbf{K})=\sum_{s} \operatorname{tr} \mathcal{O}\left(\mathbf{k}_{s}\right)=\sum_{s} \mathcal{O}^{\mathrm{unf}}\left(\mathbf{k}_{s}\right),
$$

where the symbols "Tr" and "tr" denote traces over the SC and NC orbital indices $N$ and $n$, respectively.

In practice, $\mathcal{O}^{\text {unf }}\left(\mathbf{k}_{s}\right)$ is evaluated by inserting the completeness relation in Eq. (12), leading to

$$
\mathcal{O}^{\mathrm{unf}}\left(\mathbf{k}_{s}\right)=\operatorname{tr} \mathcal{O}\left(\mathbf{k}_{s}\right)=\operatorname{Tr}\left[T\left(\mathbf{k}_{s}, \mathbf{K}\right) \mathcal{O}(\mathbf{K})\right],
$$

where

$$
\begin{aligned}
T_{M N}\left(\mathbf{k}_{s}, \mathbf{K}\right) & =\left\langle\mathbf{K} M\left|\hat{T}\left(\mathbf{k}_{s}\right)\right| \mathbf{K} N\right\rangle \\
& =\frac{1}{|\mathbf{M}|}\left[S^{\dagger}\left(\mathbf{k}_{s}, \mathbf{K}\right) S\left(\mathbf{k}_{s}, \mathbf{K}\right)\right]_{M N}
\end{aligned}
$$

and $S_{n N}\left(\mathbf{k}_{s}, \mathbf{K}\right)=\left\langle\mathbf{k}_{s} n \mid \mathbf{K} N\right\rangle$. 
Equation (14) is our generic BZ unfolding prescription. In Appendix A we verify that, when applied to the spectral operator, it gives the correct expression for the unfolded energy bands. Before considering the Berry curvature, let us discuss two properties satisfied by the unfolding procedure.

\section{Unfolding sum rule}

Summing Eq. (13) over a uniform grid in the SBZ and replacing $\sum_{\mathbf{K}}^{\mathrm{SBZ}} \sum_{s}$ with $\sum_{\mathbf{k}}^{\mathrm{NBZ}}$ we find

$$
\int_{\mathrm{SBZ}} d^{3} K \operatorname{Tr} \mathcal{O}(\mathbf{K})=\int_{\mathrm{NBZ}} d^{3} k \operatorname{tr} \mathcal{O}(\mathbf{k}) .
$$

This relation allows us to express the AHC in terms of the unfolded Berry curvature in the NBZ.

\section{Gauge invariance}

Under a unitary mixing of the SC basis states,

$$
|\mathbf{K} N\rangle \rightarrow \sum_{M}|\mathbf{K} M\rangle U_{M N}(\mathbf{K}),
$$

the matrix $T\left(\mathbf{k}_{s}, \mathbf{K}\right)$ changes in a gauge-covariant manner,

$$
T\left(\mathbf{k}_{s}, \mathbf{K}\right) \rightarrow U^{\dagger}(\mathbf{K}) T\left(\mathbf{k}_{s}, \mathbf{K}\right) U(\mathbf{K}) .
$$

Provided that $\mathcal{O}(\mathbf{K})$ is also gauge-covariant, Eq. (14) remains unchanged under the transformation by invariance of the trace. This gauge-invariance requirement will dictate which definition of a "Berry-curvature matrix" to use for unfolding purposes. (While the matrix representation (10) of most physical quantities is unique and trivially gauge-covariant, the Berry curvature is more subtle as it involves $k$-space derivatives of the state vectors.)

We note that Eq. (13) can also be obtained by evaluating the right-hand side of Eq. (14) in a basis that diagonalizes either $T\left(\mathbf{k}_{s}, \mathbf{K}\right)$ or $\mathcal{O}(\mathbf{K})$ and then using the sum rule $\sum_{s} T_{N N}\left(\mathbf{k}_{s}, \mathbf{K}\right)=1$ for the unfolding weights:

$$
\sum_{s} \mathcal{O}^{\mathrm{unf}}\left(\mathbf{k}_{s}\right)=\sum_{s, N} T_{N N}\left(\mathbf{k}_{s}, \mathbf{K}\right) \mathcal{O}_{N N}(\mathbf{K})=\operatorname{Tr} \mathcal{O}(\mathbf{K}) .
$$

\section{Unfolded Berry curvature}

Since the BZ unfolding procedure is based on matrix objects, we begin by defining a Hermitian Berry-curvature matrix $\Omega_{a b, N M}(\mathbf{K})=\Omega_{a b, M N}^{*}(\mathbf{K})$ satisfying two essential requirements: (i) It should be gauge covariant in the sense of Eq. (18) and (ii) its trace should give the quantity to be unfolded: $\operatorname{Tr} \Omega_{a b}(\mathbf{K})=\Omega_{a b}^{\text {occ }}(\mathbf{K})$.

Those requirements are fulfilled by the non-Abelian Berry curvature matrix $[2,22]$. For an insulator it reads

$$
\Omega_{a b, N M}=\partial_{a} A_{b, N M}-\partial_{b} A_{a, N M}-i\left[A_{a}, A_{b}\right]_{N M},
$$

where $\mathbf{K}$ has been dropped everywhere for brevity. Here $\partial_{a}=$ $\partial / \partial_{K_{a}}, A_{a, N M}=i\left\langle u_{N} \mid \partial_{a} u_{M}\right\rangle$ is the Berry connection matrix, and the indices $N, M$ run over the occupied states. Except for the commutator, Eq. (20) is the obvious matrix generalization of Eq. (3). The extra term does not affect the trace, but is needed to ensure gauge covariance.
For our purposes it will be convenient to recast Eq. (20) in terms of projection operators [22],

$$
\Omega_{a b, N M}=i F_{a b, N M}-i F_{b a, N M},
$$

where

$$
F_{a b, N M}=\left\langle u_{N}\left|\left(\partial_{a} \hat{P}\right) \hat{Q}\left(\partial_{b} \hat{P}\right)\right| u_{M}\right\rangle=F_{b a, M N}^{*}
$$

and $\hat{P}, \hat{Q}=\hat{\mathbb{1}}-\hat{P}$ span the occupied and unoccupied spaces, respectively. Metals can be handled by writing

$$
\hat{P}=\sum_{N, M}\left|u_{N}\right\rangle f_{N M}\left\langle u_{M}\right|,
$$

where $f_{N M}$ is the occupation matrix [23]. For insulators $\hat{P}=$ $\sum_{N}^{\text {occ }}\left|u_{N}\right\rangle\left\langle u_{N}\right|$, and a few lines of algebra show that Eq. (21) correctly reduces to Eq. (20).

With these definitions, the Berry curvatures in the original SBZ and unfolded onto the NBZ via Eq. (14) read

$$
\begin{gathered}
\Omega_{a b}^{\mathrm{occ}}(\mathbf{K})=-2 \operatorname{Im} \operatorname{Tr} F_{a b}(\mathbf{K}), \\
\Omega_{a b}^{\mathrm{unf}}\left(\mathbf{k}_{s}\right)=-2 \operatorname{Im} \operatorname{Tr}\left[T\left(\mathbf{k}_{s}, \mathbf{K}\right) F_{a b}(\mathbf{K})\right] .
\end{gathered}
$$

Equation (24) was given in Ref. [22], while Eq. (25) is a primary result of the present work.

Applying the unfolding sum rule (19) to the Berry curvature gives Eq. (7). The geometric AHC of the SC system can therefore be recast as an integral over the NBZ [Eq. (5)], in accordance with the sum rule of Eq. (16).

\section{Implementation in a Wannier basis}

In this section we describe the implementation of Eq. (25) using Wannier interpolation, which is carried out as a postprocessing step following a first-principles SC calculation. Essentially, we combine two Wannier-based methodologies: that of Refs. [10] and [23] for computing the Berry curvature and that of Ref. [4] for BZ unfolding.

In the formalism of Ref. [4] the Bloch basis orbitals are chosen as $|\mathbf{K} N\rangle=\sum_{\mathbf{R}} e^{i \mathbf{K} \cdot \mathbf{R}}|\mathbf{R} N\rangle$, where $|\mathbf{R} N\rangle$ is a Wannier function and $\mathbf{R}$ a SC lattice vector. The Wannier functions are then mapped onto the NC according to $|\mathbf{R} N\rangle \leftrightarrow|\mathbf{r} n\rangle=\mid \mathbf{R}+$ $[\mathbf{r}], n\rangle$, with a choice of $|\mathbf{M}| \mathrm{NC}$ lattice vectors $[\mathbf{r}]$ such that no two [r]'s differ by an R. Once a map has been chosen, any NC lattice vector $\mathbf{r}$ can be uniquely decomposed as $\mathbf{r}=\mathbf{R}+[\mathbf{r}]$. Setting $|\mathbf{k} n\rangle=\sum_{\mathbf{r}} e^{i \mathbf{k} \cdot \mathbf{r}}|\mathbf{r} n\rangle$ then gives [4,5]

$$
S_{n N}\left(\mathbf{k}_{s}, \mathbf{K}\right)=\left\langle\mathbf{k}_{s} n \mid \mathbf{K} N\right\rangle=e^{-i \mathbf{k}_{s} \cdot[\mathbf{r}](N)} \delta_{n, n^{\prime}(N)},
$$

which goes into the unfolding equations (15) and (14).

The expression for the unfolded Berry curvature involves several other matrix objects, which we now define borrowing the notation from Ref. [23]. The two basic objects are (omitting orbital indices)

$$
\begin{aligned}
\mathbb{H}(\mathbf{K}) & =\sum_{\mathbf{R}} e^{i \mathbf{K} \cdot \mathbf{R}}\langle\mathbf{0}|\hat{H}| \mathbf{R}\rangle, \\
\mathbb{A}_{a}(\mathbf{K}) & =\sum_{\mathbf{R}} e^{i \mathbf{K} \cdot \mathbf{R}}\left\langle\mathbf{0}\left|\hat{x}_{a}\right| \mathbf{R}\right\rangle .
\end{aligned}
$$

Diagonalization of $\mathbb{H}(\mathbf{K})$ gives the energy eigenvalues,

$$
\mathbb{H}_{J J^{\prime}}^{(\mathrm{H})}(\mathbf{K})=\left[U^{\dagger}(\mathbf{K}) \mathbb{H}(\mathbf{K}) U(\mathbf{K})\right]_{J J^{\prime}}=\epsilon_{\mathbf{K} J} \delta_{J, J^{\prime}},
$$


where the superscript $(\mathrm{H})$ stands for "Hamiltonian gauge." Next we define

$$
J_{a, J J^{\prime}}^{(\mathrm{H})}(\mathbf{K})= \begin{cases}\frac{i\left\{U^{\dagger}(\mathbf{K})\left[\partial_{a} \mathbb{H}(\mathbf{K})\right] U(\mathbf{K})\right\}_{J J^{\prime}}}{\epsilon_{\mathbf{K} J^{\prime}}-\epsilon_{\mathbf{K} J}} & \text { if } J^{\prime} \neq J, \\ 0 & \text { if } J^{\prime}=J,\end{cases}
$$

and $J_{a}=U J_{a}^{(\mathrm{H})} U^{\dagger}$. This matrix will only appear in the combinations $J_{a}^{-}=f J_{a} g$ and $J_{a}^{+}=g J_{a} f$, where $f$ is the occupation matrix introduced in Eq. (23), and $g=1-f$. With these definitions, the unfolded curvature in the Wannier basis becomes (see derivation in Appendix B)

$$
\begin{aligned}
\Omega_{a b}^{\mathrm{unf}}\left(\mathbf{k}_{s}\right)= & \operatorname{Re} \operatorname{Tr}\left[T f\left(\partial_{a} \mathbb{A}_{b}-\partial_{b} \mathbb{A}_{a}\right) f\right] \\
& +2 \operatorname{Im} \operatorname{Tr}\left[T f \mathbb{A}_{a} f \mathbb{A}_{b} f\right] \\
& -2 \operatorname{Im} \operatorname{Tr}\left[T\left(f \mathbb{A}_{a} J_{b}^{+}+J_{a}^{-} \mathbb{A}_{b} f+J_{a}^{-} J_{b}^{+}\right)\right] .
\end{aligned}
$$

Equation (31) is our second important result. It expresses the unfolded Berry curvature at a point $\mathbf{k}_{s}$ in the NBZ in terms of the matrix $T\left(\mathbf{k}_{s}, \mathbf{K}\right)$ given by Eqs. (15) and (26), and additional matrices defined at the folded point $\mathbf{K}$ in the SBZ. Those other matrices can be computed from a knowledge of the Hamiltonian and position-operator matrix elements in the Wannier basis, which are then Fourier transformed into $\mathbb{H}(\mathbf{K})$ and $\mathbb{A}_{a}(\mathbf{K})$ via Eqs. (27) and (28). Diagonalization of $\mathbb{H}(\mathbf{K})$ [Eq. (29)] provides the energy eigenvalues and rotation matrices used to compute $f(\mathbf{K})$ and $J_{a}^{ \pm}(\mathbf{K})$ [23]. Note that the needed derivatives $\partial_{a} \mathbb{H}(\mathbf{K})$ and $\partial_{b} \mathbb{A}_{a}(\mathbf{K})$ are easily obtained by differentiating Eqs. (27) and (28).

It is instructive to consider the trivial unfolding scenario where the NC and the SC are the same. Then $T$ becomes the identity matrix, the second term in Eq. (31) vanishes since $\operatorname{Im} \operatorname{Tr}\left[\mathbb{A}_{a} f \mathbb{A}_{b} f\right]=0$, and $\boldsymbol{\Omega}^{\text {unf }}(\mathbf{k})$ correctly reduces to Eq. (51) of Ref. [23] for $\boldsymbol{\Omega}^{\text {occ }}(\mathbf{k})$.

\section{COMPUTATIONAL DETAILS}

Plane-wave pseudopotential calculations were carried out for bcc $\mathrm{Fe}$, bcc Co, and an Fe-Co ordered alloy with the $\mathrm{Fe}_{3} \mathrm{Al}$ structure [24]. The experimental lattice constant $a=5.42 \mathrm{bohr}$ of bcc Fe was used in all cases to facilitate comparisons, and the magnetization was set along the [001] direction.

The calculations were performed with the PWSCF code from the QUANTUM-ESPRESSO package [25], in a noncollinear spin framework with fully relativistic norm-conserving pseudopotentials generated from parameters similar to those in Ref. [10]. An energy cutoff of $120 \mathrm{Ry}$ was used for the plane-wave expansion of the wave functions, and exchange and correlation effects were treated within the PBE generalized-gradient approximation [26].

In the case of bcc $\mathrm{Fe}$ and bcc $\mathrm{Co}$, the self-consistent total energy calculations were done with a $16 \times 16 \times 16$ Monkhorst-Pack mesh for the BZ integration, while for the non-self-consistent calculation a $10 \times 10 \times 10$ mesh was used, and the 28 lowest bands were calculated. In the case of $\mathrm{Fe}_{3} \mathrm{Co}$ the $\mathrm{BZ}$ integration meshes were $12 \times 12 \times 12$ and $10 \times 10 \times 10$ for the self-consistent and band-structure calculations, respectively, and the 112 lowest bands were calculated. A Fermi smearing of $0.02 \mathrm{Ry}$ was used in all self-consistent calculations.
For each material, 18 spinor Wannier functions per atom were then constructed using WANNIER90 [27]. Atom-center $s$-, $p$-, and $d$-like trial orbitals were used for the initial projection step, followed by an iterative procedure to select an optimal "disentangled" subspace [28], using the same inner and outer energy windows as in Ref. [10]. At variance with that work, no minimization of the spread functional was done to further improve the localization properties of the "projected" Wannier functions [29]. This was done to keep the Wannier functions of $\mathrm{Fe}_{3} \mathrm{Co}$ as similar as possible to those of bcc $\mathrm{Fe}$, as required by the Wannier-based unfolding scheme [4].

In the next section we show results for the energy bands and Berry curvature of the ordered $\mathrm{Fe}_{3} \mathrm{Co}$ alloy unfolded onto the NBZ of bcc Fe. For comparison purposes, we also show the energy bands and Berry curvatures of pure Fe and of the VCA alloy computed directly in the NBZ. Following Ref. [15], we have implemented the VCA in the basis of projected Wannier functions, by linearly mixing the Hamiltonian matrix elements of bcc Fe and bcc Co. Since the Wannier interpolation of the Berry curvature also requires the position-operator matrix elements [10], we modified them accordingly.

In all cases, with and without unfolding, we plot the Bloch spectral function instead of the energy bands. To generate the plots we use the method of Ref. [13], adapted to display the spin polarization $\left\langle S_{z}\right\rangle$ as a color code. A similar procedure is used to plot the intersections of the (unfolded) Fermi surface with a plane in the NBZ. For simplicity, we continue to use the expressions "energy bands" and "Fermi surface intersections" (or "Fermi lines") when referring to the figures.

\section{RESULTS}

We have selected Fe-Co, a substitutional alloy based on the bcc structure, as a test case for the Berry-curvature unfolding procedure. We focus on a composition of $25 \% \mathrm{Co}$, using the $\mathrm{Fe}_{3} \mathrm{Al}$ ordered structure as a representative configuration [24]. This structure is of the Heusler $X_{2} Y Z$ type, with $X=Y=\mathrm{Fe}$ and $Z=\mathrm{Co}$ (pure bcc Fe corresponds to $X=Y=Z=\mathrm{Fe}$ ). The Bravais lattice is fcc with a four-atom basis, so that the energy bands live in a folded BZ which is four times smaller than the NBZ of bcc Fe. This makes a direct comparison with the band structure of bcc Fe rather difficult, and typically only the densities of states are compared [24].

Information about the $k$-space distribution of the electron states in the alloy can be recovered by plotting the energy bands unfolded onto the NBZ [Eq. (A3)]. The result, shown in the bottom panel of Fig. 1, strongly resembles the bands of bcc Fe in the top panel. The influence of the Co atoms is clearly visible in certain regions of the $(\mathbf{k}, E)$ plane, in the form of "broken bands" and "ghost bands." Overall, the effects of alloying are most pronounced for the narrow $d$ bands crossing the Fermi level.

We now turn to the $k$-space Berry curvature, Eqs. (2) and (6), and begin by recalling its salient features in crystalline metallic ferromagnets [8-10]. In this class of materials the Berry curvature is induced by the combined effect of exchange splitting and spin-orbit coupling, which together break timereversal symmetry in the orbital wave functions. $\boldsymbol{\Omega}^{\text {occ }}(\mathbf{k})$ is characterized by strong, sharp features which are concentrated around the Fermi surface in regions where occupied and empty 


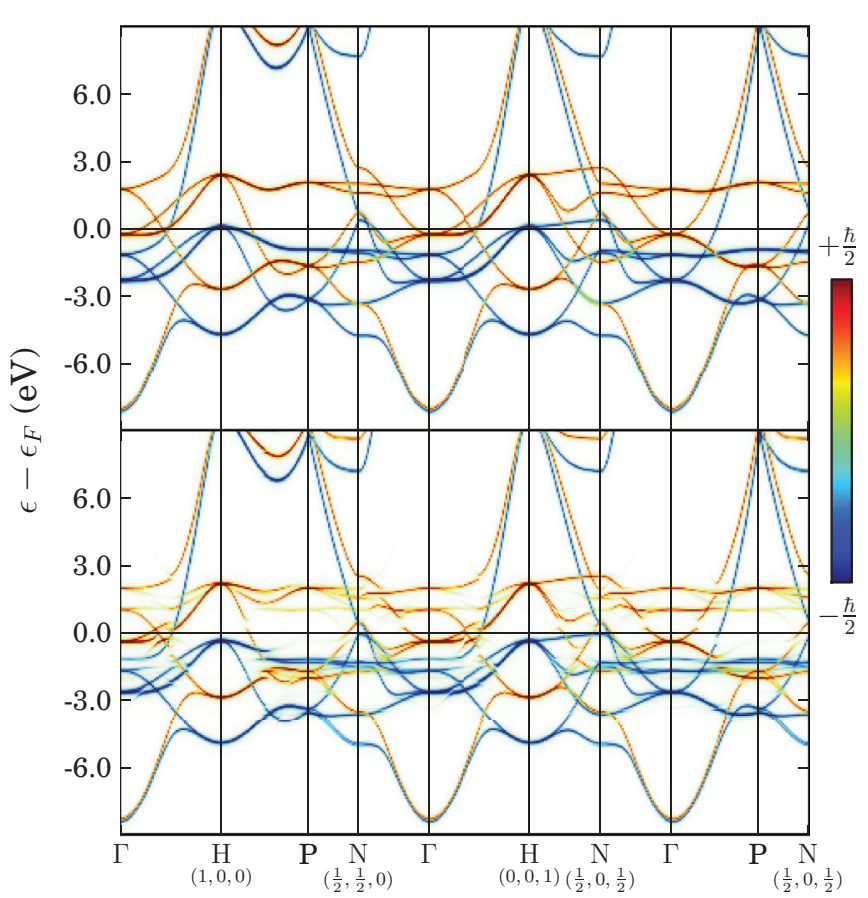

FIG. 1. (Color online) (Top) Energy bands of bcc Fe. (Bottom) Energy bands of the fcc $\mathrm{Fe}_{3} \mathrm{Co}$ alloy unfolded onto the $\mathrm{BZ}$ of bcc Fe. Colors indicate the spin polarization $\left\langle S_{z}\right\rangle$ of the states. The points labeled $\mathrm{P}$ all have coordinates $\left(\frac{1}{2}, \frac{1}{2}, \frac{1}{2}\right)$. The bands of the bcc $\left\langle\mathrm{Fe}_{3} \mathrm{Co}\right\rangle$ virtual crystal (not shown) are almost indistinguishable from those of bcc Fe, except for a shift in the Fermi level.

bands come in close contact and become strongly coupled by spin orbit. This is illustrated for bcc Fe in Fig. 2 (top), which displays the energy bands near the Fermi level and the Berry curvature, along the $\Gamma-\mathrm{H}-\mathrm{P}$ path. The spiky features rise above a smooth, low-intensity background which is visible in the heat-map plot of the Berry curvature over the $k_{y}=0$ plane [Fig. 3 (top)].

In order to understand how alloying with Co disturbs the Berry curvature, we first consider the effects in the VCA, that is, for a bcc crystal composed of "averaged" $\left\langle\mathrm{Fe}_{3} \mathrm{Co}\right\rangle$ atoms. Since $\mathbf{k}$ remains a good quantum number in the NBZ, the energy bands and Berry curvature can be obtained in the usual manner (without unfolding), and are shown in Fig. 2 (middle). The bands are quite similar to those of bcc Fe, and the main effect of alloying is an upward shift of the Fermi level. This leads to significant changes in the Berry curvature: For example, the strong peak along H-P is completely suppressed, since the two majority bands involved are now both occupied. Only some very low-intensity features remain along $\Gamma-\mathrm{H}$ (note the difference in the Berry curvature scales between the panels in Fig. 2).

Comparing the heatmaps in Figs. 3 (top) and 3 (middle) we again see significant differences in the Berry-curvature distribution due to the shift in the Fermi level across the narrow $d$ bands. In both cases the Berry curvature is concentrated in regions where there are weak avoided crossings between two Fermi lines, which can be of opposite-spin character or of like-spin character.
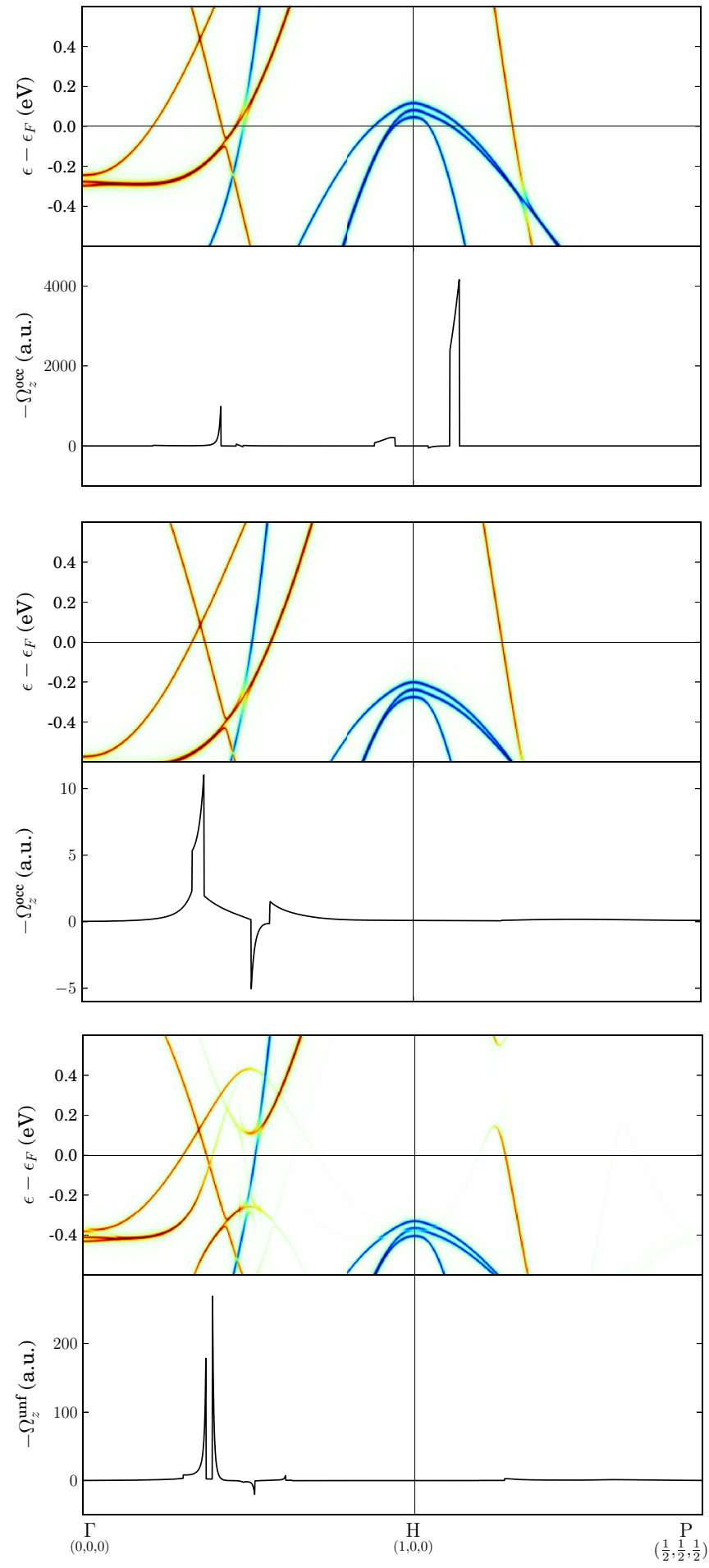

FIG. 2. (Color online) Energy bands color coded by the spin polarization $\left\langle S_{z}\right\rangle$ and Berry curvature summed over the occupied states, plotted along the path $\Gamma$-H-P. (Top) bcc Fe. (Middle) bcc $\left\langle\mathrm{Fe}_{3} \mathrm{Co}\right\rangle$ virtual crystal. (Bottom) fcc $\mathrm{Fe}_{3} \mathrm{Co}$ alloy, using $\mathrm{BZ}$ unfolding.

Missing from the VCA description of the alloy are the effects brought about by the reduced translational order, which are the main focus of this work. Their influence on the band structure was revealed by plotting the unfolded bands of fcc $\mathrm{Fe}_{3} \mathrm{Co}$ in Fig. 1. In order to see how the Berry curvature is 

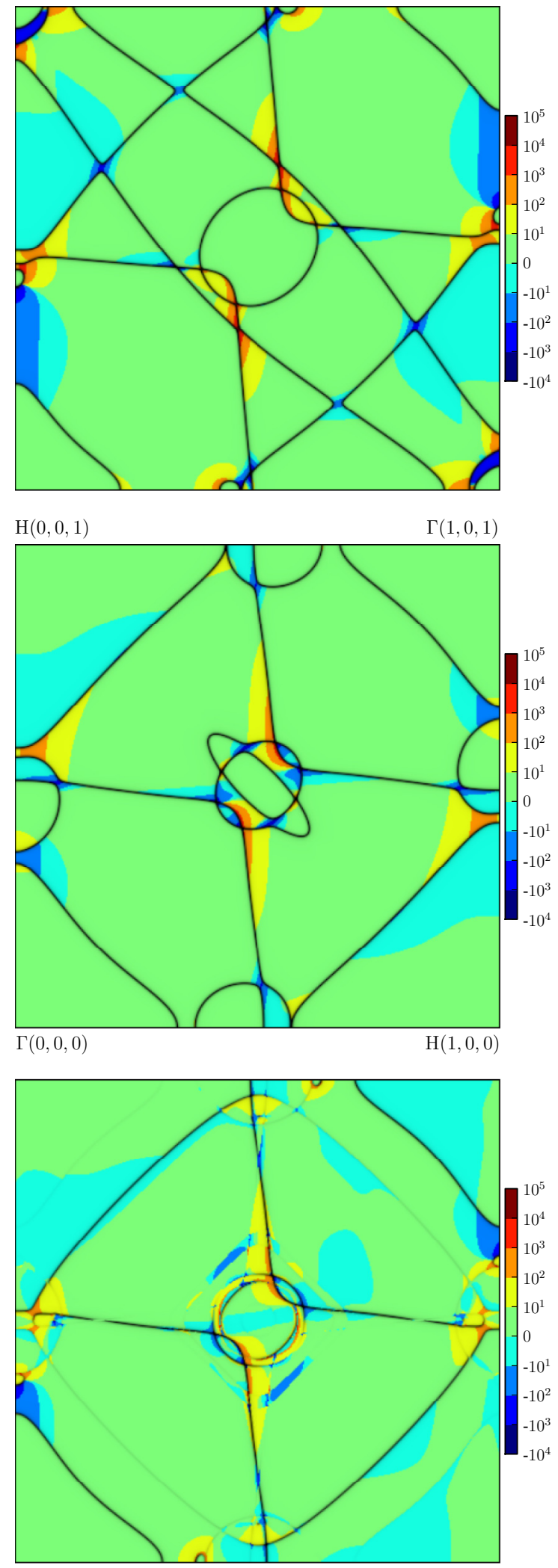

FIG. 3. (Color online) Heat map of the Berry curvature in the plane $k_{y}=0$, in atomic units (note the $\log$ scale). The lines of intersection between the Fermi surface and the plane are also shown. The top and middle panels show $-\Omega_{z}^{\text {occ }}$ for bcc Fe and bcc $\left\langle\mathrm{Fe}_{3} \mathrm{Co}\right\rangle$ respectively, and the bottom panel shows $-\Omega_{z}^{\text {unf }}$ for fcc $\mathrm{F}_{3} \mathrm{Co}$. affected, we plot together in Fig. 2 (bottom) the two unfolded quantities, energy bands and Berry curvature.

Compared to the VCA results in Fig. 2 (middle) the Fermi level has not moved appreciably, and the bigger changes are in the bands themselves, especially in the minority states near the Fermi level. The Berry curvature displays two contiguous strong peaks along $\Gamma-\mathrm{H}$. They are associated with spectral features which have been greatly modified with respect to the VCA calculation, namely, a pair of minority bands with a weak avoided crossing just below the Fermi level. As the upper band rises above $\epsilon_{F}$ on either side of the crossing, a Berry-curvature peak suddenly develops and then quickly drops as the separation between the two bands increases. Plots along other high-symmetry lines show similar features. We conclude that the intuitive interband-coupling interpretation of the Berry curvature based on Eq. (6) carries over to the unfolded curvature, now in terms of the unfolded bands. Further confirmation of this comes from inspecting the unfolded Fermi lines and Berry curvature across the $k_{y}=0$ plane in Fig. 3 (bottom). Overall, they resemble those of the VCA crystal, but with some distortions. As before, the Berry curvature is concentrated in regions where two Fermi lines approach one another.

We conclude by evaluating the AHC of the three systems from Eqs. (1) and (4). The results were carefully converged with respect to $k$-point sampling $[9,10]$, using dense uniform meshes which were adaptively refined around points where the Berry curvature exceeded a threshold magnitude of $27.98 \AA^{2}$. Uniform (adaptive) meshes of up to $350 \times 350 \times 350(13 \times$ $13 \times 13)$ in the $\mathrm{NBZ}$ were used for $\mathrm{Fe}$ and $\left\langle\mathrm{Fe}_{3} \mathrm{Co}\right\rangle$. For $\mathrm{Fe}_{3} \mathrm{Co}$ the densest uniform (adaptive) mesh in the SBZ was $250 \times 250 \times 250(11 \times 11 \times 11)$. The converged AHC values are $758 \mathrm{~S} / \mathrm{cm}$ for bcc $\mathrm{Fe}, 452 \mathrm{~S} / \mathrm{cm}$ for bcc $\left\langle\mathrm{Fe}_{3} \mathrm{Co}\right\rangle$, and $473 \mathrm{~S} / \mathrm{cm}$ for fcc $\mathrm{Fe}_{3} \mathrm{Co}$. We comment on these numbers shortly.

\section{DISCUSSION AND OUTLOOK}

As illustrated by our calculations, impurities modify the interband couplings responsible for the intrinsic $\mathrm{AHC}$ in perfectly ordered crystals. In the context of SC calculations it is very natural to combine the putative intrinsic contribution of Eq. (1) with those disorder corrections into a single geometric contribution, Eq. (4), which is a gauge-invariant property of the disordered electronic ground state. In Chern insulators, where the AHE is quantized for topological reasons (QAHE), the disorder corrections cancel out upon taking the integral in Eq. (4) [2]. In metals the AHE is not quantized, and disorder gives a net geometric contribution on top of the intrinsic one.

In the same way that the intrinsic AHC can be viewed as the dc limit of the interband conductivity of the pristine crystal [1], the geometric AHC corresponds to the dc limit of the interband conductivity of a SC with disorder, whose "bands" are defined in the folded BZ. For disorder of the cellular type, the familiar representation in terms of a Berry curvature in the normal BZ can be restored by means of the unfolded Berry curvature $\boldsymbol{\Omega}^{\text {unf }}(\mathbf{k})$ of Eq. (25), leading to Eq. (5), which has the same form as Eq. (1). 
In pristine crystals the geometric $\mathrm{AHC}$ reduces to the intrinsic contribution. It therefore retains the essential features of the intrinsic theory of the AHE, while at the same time addressing the main criticism that it originally faced, namely, "the complete absence of scattering from disorder in the derived Hall response contribution" [1].

First-principles calculations based on Eq. (1) have been carried out for a number of ferromagnetic metals and alloys, often finding reasonably good agreement with experiment: Examples include $\mathrm{SrRuO}_{3}$ [8], bcc Fe [9,10], hcp Co [30,31], and $\mathrm{CuCr}_{2} \mathrm{Se}_{4-x} \mathrm{Br}_{x}$ [17]. A large discrepancy between $a b$ initio theory and experiment in the case of fcc $\mathrm{Ni}$ [30] was eventually resolved by carrying out more sophisticated GGA + U calculations [32].

Given the success of the intrinsic theory, one should be cautious about introducing modifications. The calculations presented in this work are reassuring in that regard: Most of the large change in the calculated AHC between pure bcc $\mathrm{Fe}$ and the Fe-Co alloy is recovered at the VCA "intrinsic" level from the band-filling effect, while "scattering" effects from the reduced translational order in the fcc cell give some corrections, without dramatically changing the result. The same conclusion can be drawn from comparing the panels in Fig. 3.

The system we have studied is, of course, a very crude model for a real disordered alloy. Calculations using larger SCs with more realistic descriptions of disorder will be needed to make detailed comparisons between the (unfolded) Berry curvature and $\mathrm{AHC}$ of a disordered crystal or alloy and those of the pristine reference crystal. For example, it seems plausible that disorder-induced contributions will be smoothened out compared to the sharp features seen in Figs. 2 (bottom) and 3 (bottom). The Wannier-based SC methodology of Ref. [13] seems particularly well suited for such studies.

It would be desirable to clarify which scattering contributions are included in the geometric AHC. We give a discussion based on the Kubo-Greenwood conductivity formula [33,34], written here for $\omega=0$,

$$
\sigma_{a b}=\frac{i \hbar e^{2}}{N V} \sum_{\mathbf{K} J J^{\prime}} \frac{f_{J^{\prime}}-f_{J}}{\epsilon_{J^{\prime}}-\epsilon_{J}} \frac{\left\langle J\left|\hat{v}_{a}\right| J^{\prime}\right\rangle\left\langle J^{\prime}\left|\hat{v}_{b}\right| J\right\rangle}{\epsilon_{J^{\prime}}-\epsilon_{J}-i \eta},
$$

where $V$ is the $\mathrm{SC}$ volume and the $\mathrm{SBZ}$ is sampled over $N$ points K. The full AHC, the sum of intrinsic, skewscattering, and side-jump contributions, can be calculated as the antisymmetric part of Eq. (32). Let us recall the role played by the parameter $\eta$ in SC calculations. For a finite volume $V$ the energy levels at fixed $\mathbf{K}$ are discrete, and absorption becomes impossible at frequencies smaller than the level spacing. It is for this reason that when calculating the residual resistivity $\rho_{x x} \simeq 1 / \sigma_{x x}$ one must use a level broadening $\eta(V)$ greater than the mean level spacing at $\epsilon_{F}$ [35]. Similar considerations may be relevant for $\sigma_{x y}$, particularly when trying to recover the skew-scattering contribution, which scales as $\sigma_{x x}$ and has a similar physical origin [1].

This analysis suggests that $\sigma_{x y}^{\text {geom }}$, which is obtained from Eq. (32) by taking the $\eta \rightarrow 0^{+}$limit at finite $V$, may not include skew scattering. We also note that since the longitudinal conductivity $\sigma_{x x}$ vanishes in that limit, $\sigma_{x y}^{\text {geom }}$ corresponds to the dissipationless part of $\sigma_{x y}$, and this is precisely how the sum of the intrinsic and side-jump contributions is defined [1] and measured at low temperatures [36].

Leaving aside matters of definition and interpretation, our gauge-invariant procedure for unfolding the Berry curvature from SC calculations seems useful in its own right as an analysis tool complementary to the unfolding of energy bands. The $k$-space Berry curvature induced by interband coherence effects has emerged as a powerful paradigm to describe the AHE [1,2], and the methods developed in this work seamlessly incorporate scattering from disorder into the picture.

In closing, we mention that the $\mathrm{BZ}$ unfolding procedure can be readily applied to other $k$-space quantities which take the form of traces over gauge-covariant matrices. Examples include the occupation numbers $n(\mathbf{K})=\operatorname{Tr} f(\mathbf{K})$, the integrand of the $k$-space orbital magnetization formula [22,23], and the quantum metric [29].

\section{ACKNOWLEDGMENTS}

This work was supported by Grants No. MAT2012-33720 from the Ministerio de Economía y Competitividad (Spain), No. CIG-303602 from the European Commission, and ONR Grant No. N00014-12-1-1041 (USA).

\section{APPENDIX A: UNFOLDED ENERGY BANDS}

The spectral operator $(E+i \eta-\hat{H})^{-1}$ projected onto the Bloch space at $\mathbf{K}$ reads, in the SC eigenstate basis,

$$
\hat{G}_{\mathbf{K}}(E+i \eta)=\sum_{J} \frac{|\mathbf{K} J\rangle\langle\mathbf{K} J|}{E+i \eta-\epsilon_{\mathbf{K} J}} .
$$

The K-resolved density of states (Bloch spectral function) consists of sharp peaks in the SBZ, corresponding to the "folded" energy bands:

$$
\begin{aligned}
D_{\mathbf{K}}(E) & =-\frac{1}{\pi} \lim _{\eta \rightarrow 0^{+}} \operatorname{Im} \operatorname{Tr} G_{\mathbf{K}}(E+i \eta) \\
& =\sum_{J} \delta\left(E-\epsilon_{\mathbf{K} J}\right) .
\end{aligned}
$$

Applying the unfolding prescription of Eq. (14) to the operator $\hat{\mathcal{O}}=(-1 / \pi) \hat{G}_{\mathbf{K}}(E+i \eta)$ we find

$$
\begin{aligned}
D_{\mathbf{k}_{s}}^{\mathrm{unf}}(E) & =\lim _{\eta \rightarrow 0^{+}} \operatorname{Im} \operatorname{tr} \mathcal{O}\left(\mathbf{k}_{i}\right) \\
& =\sum_{J} T_{J J}\left(\mathbf{k}_{s}, \mathbf{K}\right) \delta\left(E-\epsilon_{\mathbf{K} J}\right) .
\end{aligned}
$$

This is the known expression for the unfolded Bloch spectral function [4], where

$$
T_{J J}\left(\mathbf{k}_{s}, \mathbf{K}\right)=\frac{1}{|\mathbf{M}|} \sum_{n}\left|\left\langle\mathbf{k}_{s} n \mid \mathbf{K} J\right\rangle\right|^{2}
$$

is the spectral weight of $|\mathbf{K} J\rangle$ at $\mathbf{k}_{s}$. (The factor of $1 /|\mathbf{M}|$ on the right-hand side is absent when adopting the normalization convention of Ref. [4].)

\section{APPENDIX B: DERIVATION OF Eq. (31)}

In this Appendix we derive Eq. (31) for $\Omega_{a b}^{\mathrm{unf}}\left(\mathbf{k}_{s}\right)$ starting from Eq. (25). Following Ref. [23], we adopt a notation 
where matrix objects written with a double staff, such as $\mathbb{A}_{N M}(\mathbf{K})=i\left\langle u_{\mathbf{K} N} \mid \partial_{a} u_{\mathbf{K} M}\right\rangle$ in Eq. (28), are defined over the space spanned by the Wannier functions, which for metals typically contains some low-lying empty states in addition to all the occupied states [28]. Instead, objects with a single staff such as $A_{N M}(\mathbf{K})$ in Eq. (20) are defined over the occupied subspace. So, for example, we define (dropping $\mathbf{K}$ everywhere) $\hat{\mathbb{P}}=\sum_{N}\left|u_{N}\right\rangle\left\langle u_{N}\right|, \hat{\mathbb{Q}}=\hat{\mathbb{1}}-\hat{\mathbb{P}}$, and $\mathbb{F}_{a b, N M}=$ $i\left\langle\partial_{a} u_{N}|\hat{\mathbb{Q}}| \partial_{b} u_{M}\right\rangle$ as counterparts to $\hat{P}, \hat{Q}$, and $F_{a b, N M}$ in Eqs. (22) and (23).

We further condense our notation by dropping indices $N$ and $M$, e.g., $\mathbb{P}=|u\rangle\langle u|$ and $\hat{P}=|u\rangle f\langle u|$. We use the relations [23]

$$
\left(\partial_{a} \hat{P}\right) \hat{Q}=|u\rangle f\left\langle\partial_{a} u|\hat{\mathbb{Q}}+i| u\right\rangle f\left(\mathbb{A}_{a}+J_{a}\right) g\langle u|
$$

and [compare with Eqs. (20) and (21)]

$$
i \mathbb{F}_{a b}-i \mathbb{F}_{b a}=\partial_{a} \mathbb{A}_{b}-\partial_{b} \mathbb{A}_{a}-i\left[\mathbb{A}_{a}, \mathbb{A}_{b}\right] \text {. }
$$

Expanding Eq. (22) with the help of Eq. (B1), we find

$F_{a b}=f \mathbb{F}_{a b} f+f \mathbb{A}_{a} g \mathbb{A}_{b} f+J_{a}^{-} \mathbb{A}_{b} f+f \mathbb{A}_{a} J_{b}^{+}+J_{a}^{-} J_{b}^{+}$.

Writing Eq. (25) as $\Omega_{a b}^{\mathrm{unf}}=i \operatorname{Tr}\left\{T\left[F_{a b}-F_{b a}\right]\right\}$, inserting Eq. (B3) and then using Eq. (B2), we arrive at Eq. (31).
[1] N. Nagaosa, J. Sinova, S. Onoda, A. H. MacDonald, and N. P. Ong, Rev. Mod. Phys. 82, 1539 (2010).

[2] D. Xiao, M.-C. Chang, and Q. Niu, Rev. Mod. Phys. 82, 1959 (2010).

[3] N. E. Cusack, The Physics of Structurally Disordered Matter (Adam Hilger, Bristol, 1987).

[4] W. Ku, T. Berlijn, and C.-C. Lee, Phys. Rev. Lett. 104, 216401 (2010).

[5] T. Berlijn, Ph.D. thesis, Stony Brook University, 2011.

[6] V. Popescu and A. Zunger, Phys. Rev. B 85, 085201 (2012).

[7] P. B. Allen, T. Berlijn, D. A. Casavant, and J. M. Soler, Phys. Rev. B 87, 085322 (2013).

[8] Z. Fang, N. Nagaosa, K. Takahashi, A. Asamitsu, R. Matthieu, T. Ogasawara, H. Yamada, M. Kawasaki, Y. Tokura, and K. Terakura, Science 302, 92 (2003).

[9] Y. Yao, L. Kleinman, A. H. MacDonald, J. Sinova, T. Jungwirth, D.-S. Wang, E. Wang, and Q. Niu, Phys. Rev. Lett. 92, 037204 (2004).

[10] X. Wang, J. R. Yates, I. Souza, and D. Vanderbilt, Phys. Rev. B 74, 195118 (2006).

[11] C. M. Hurd, The Hall Effect in Metals and Alloys (Plenum, New York, 1972).

[12] S. Baroni, S. de Gironcoli, and P. Giannozzi, Phys. Rev. Lett. 65, 84 (1990).

[13] T. Berlijn, D. Volja, and W. Ku, Phys. Rev. Lett. 106, 077005 (2011).

[14] T. Berlijn, P. J. Hirschfeld, and W. Ku, Phys. Rev. Lett. 109, 147003 (2012).

[15] J. Liu and D. Vanderbilt, Phys. Rev. B 88, 224202 (2013).

[16] S. Lowitzer, D. Ködderitzsch, and H. Ebert, Phys. Rev. Lett. 105, 266604 (2010)

[17] Y. Yao, Y. Liang, D. Xiao, Q. Niu, S.-Q. Shen, X. Dai, and Z. Fang, Phys. Rev. B 75, 020401 (2007).
[18] T. Jungwirth, Q. Niu, and A. H. MacDonald, Phys. Rev. Lett. 88, 207208 (2002).

[19] H. Zhang, S. Blügel, and Y. Mokrousov, Phys. Rev. B 84, 024401 (2011).

[20] E. Prodan, T. L. Hughes, and B. A. Bernevig, Phys. Rev. Lett. 105, 115501 (2010).

[21] Y.-F. Zhang, Y.-Y. Y. anf Y. Ju, L. Sheng, R. Shen, D.-N. Sheng, and D.-Y. Xing, Chin. Phys. B 22, 117312 (2013).

[22] D. Ceresoli, T. Thonhauser, D. Vanderbilt, and R. Resta, Phys. Rev. B 74, 024408 (2006).

[23] M. G. Lopez, D. Vanderbilt, T. Thonhauser, and I. Souza, Phys. Rev. B 85, 014435 (2012).

[24] K. Schwarz, P. Mohn, and J. Kübler, J. Phys. F: Met. Phys. 14, 2659 (1984).

[25] P. Giannozzi et al., J. Phys.: Condens. Matter 21, 395502 (2009).

[26] J. P. Perdew, K. Burke, and M. Ernzerhof, Phys. Rev. Lett. 77, 3865 (1996).

[27] A. A. Mostofi, J. R. Yates, Y.-S. Lee, I. Souza, D. Vanderbilt, and N. Marzari, Comput. Phys. Commun. 178, 685 (2008).

[28] I. Souza, N. Marzari, and D. Vanderbilt, Phys. Rev. B 65, 035109 (2001).

[29] N. Marzari and D. Vanderbilt, Phys. Rev. B 56, 12847 (1997).

[30] X. Wang, D. Vanderbilt, J. R. Yates, and I. Souza, Phys. Rev. B 76, 195109 (2007).

[31] E. Roman, Y. Mokrousov, and I. Souza, Phys. Rev. Lett. 103, 097203 (2009).

[32] H.-R. Fuh and G.-Y. Guo, Phys. Rev. B 84, 144427 (2011).

[33] K. Nomura and A. H. MacDonald, Phys. Rev. Lett. 98, 076602 (2007).

[34] A. Shitade and N. Nagaosa, J. Phys. Soc. Jpn. 81, 083704 (2012).

[35] R. H. Brown, P. B. Allen, D. M. Nicholson, and W. H. Butler, Phys. Rev. Lett. 62, 661 (1989).

[36] Y. Tian, L. Ye, and X. Jin, Phys. Rev. Lett. 103, 087206 (2009). 